\title{
Research on the Effect of Pentosans from Wheat Bran on Bread Quality Baolan Bai ${ }^{1, a}$ Baiying $\mathrm{Cao}^{2, b}$ Xiue Sun ${ }^{3, \mathrm{c}}$ \\ 123 Jilin Engineering Normal University, Changchun, Jilin, China, 130052 \\ aemail, ${ }^{b}$ email, ${ }^{\mathrm{c} e m a i l,}$
}

Keywords: Effect, Pentosans, Wheat Bran, Bread Quality

\begin{abstract}
Wheat flour were prepared to make aqueous pentosan and alkali soluble pentosan and then they were added to wheat flour in different proportions to conduct bread baking experiments to study its effect on the quality of the bread baking.
\end{abstract}

\section{Introduction}

Early last century, with the chemical industry on the quality of grain wheat depth and found that in addition to the main constituent components of wheat - Protein and Starch has an important influence on wheat quality, but at a level of less and has a high viscosity component wheat quality also plays a very important role. In 1927, Hoffmann et al bread from wheat flour isolated from this kind of non-starch polysaccharides having a higher viscosity, the study confirmed that it is mainly composed of pentoses (arabinose and xylose) composed, they put their name pentosan

Pentosan mainly has the following important features two aspects: First, have a high water absorption and water holding capacity, higher viscosity dispersed in the aqueous phase was formed; the second is having a small amount in the case of the presence of an oxidizing agent oxidation crosslinking properties, which makes it rheological properties and bread quality has a very significant impact, mainly in the following areas: the impact ${ }^{1}$ dough water absorption is generally believed that dough water absorption depends protein content of the flour and starch damage, but studies have found a high degree of hydration pentosan capability although the proportion in the flour accounted for the low (dry weight flour accounted for $1.5 \%$ to $3 \%$ ), but the amount of water absorption of dough there very important influence in the dough formation, pentosan moisture absorbed $23 \%$ of the total amount of water absorption of the dough, therefore, pentosan dough water absorption and dough moisture distribution is an important regulator. Affect dough rheology. pentosans may be crosslinked oxidation, can enhance the cohesion of the dough, increased flexibility, extensibility decreased for improving poor silty flour, in addition to adding gluten protein, added the right amount of pentosan can achieve good results. Add the proper amount of pentosan to flour can increase the volume of bread, in addition, pentosan also delay the aging of bread, to extend its shelf life.

Past studies on pentosan mainly in the endosperm of wheat and rye pentosan on bread quality, as pentosans on dough properties and bread baking quality of non-endosperm sources have not been reported. Wheat pentosan mainly in the outer portion of wheat that is a byproduct of wheat processing - wheat bran, the amount of $20 \%$ or more of the present experiment, bran, pentosan prepared to study the properties of dough and bread quality.

\section{Research on Pentosan}

Many cereals are pentosan exist, such as wheat, rye, barley, oats, rice, sorghum and the like. Pentosan content in wheat grain is about $5 \%-8 \%$, pentosan content in wheat flour is generally about $2 \%-3 \%$ and the content of pentosan bran is about $20 \%$. Pentosan content in wheat, although very little, but the quality of wheat has played a very important role, such as pentosan Hardness wheat processing quality of wheat, dough viscosity, rheological properties, baking quality and starch retrogradation and so on.

Pentosan because it has a higher water absorption properties of water retention, high viscosity characteristics, good emulsion stability and oxidation crosslinking properties make quality Dough 
Properties and flour products have a great impact, nearly a century and it has been a hot research community cereal Chemistry. Through the study found, pentosan Dough Properties and Bread Quality focused on the following aspects: 1, pentosan increase the amount of water absorption of the dough. 2, pentosan crosslinking can occur oxidation, the gluten network to form larger structures, can enhance the cohesion of the dough, increased flexibility, extensibility decreased, thereby improving the rheological properties of dough. 3. An appropriate amount of wheat flour pentosans to bread volume can be increased to improve the texture of the internal structure of the bread, addition, pentosan also effectively delay aging of bread, to extend their shelf life. Pentosan past research focused on the characteristics of the endosperm of wheat and rye pentosan and its impact on the quality of baking bread, as bread baking properties and quality of non-endosperm sources pentosan dough is very less. Because wheat pentosan mainly in the outer portion of wheat that is a byproduct of wheat processing - wheat bran, the amount of $20 \%$ or more. Therefore, this experiment was prepared from wheat bran pentosan wheat on bread baking quality bran.

\section{Preparation of Pentosan with Wheat Bran}

Crushed and sieved wheat bran, adding a certain amount of distilled water, adjusting the $\mathrm{pH}$ and adding an appropriate amount a- amylase, heated to $95^{\circ} \mathrm{C}$, and shaken incubated $1 \mathrm{~h}$, then cooled to room temperature, centrifuged, the residue was collected and dried to obtain the removal of starch wheat bran (DS - WB). DS - WB solution was extracted with 0 . $2 \mathrm{NNaOH}$ at normal temperature $2 \mathrm{~h}$, followed by centrifugation, the supernatant was concentrated by centrifugation after $\mathrm{pH}$ was adjusted with glacial acetic acid, centrifuged, and the supernatant was centrifuged, adding 4 volumes of ethanol at $4{ }^{\circ} \mathrm{C}$ approximately to stand overnight in a refrigerator, the supernatant was decanted, the precipitate was collected, dissolved a certain amount of water was added, freeze-drying, is water soluble pentosan bran (WB - WSP).

$\mathrm{PH}$ adjusted with acetic acid precipitated after centrifugation, respectively a- amylase, protease, and then precipitated by centrifugation, after centrifugation, adding a certain amount of distilled water, freeze-dried, to give a water-insoluble pentosan bran (WB - WIP).

\section{Composition Component Analysis of Pentosan}

Pentosan determination, phloroglucinol method; determination of protein content, micro-Kjeldahl method; determination of ferulic acid, 325nm spectrophotometry: monosaccharide composition analysis, gas chromatography; determination of uronic acid content, improved carbazole method. the composition prepared from wheat bran pentosan components were analyzed, which shows that pentosan a purity of about $60 \%$, in addition, it also contains about $15 \%$ protein and other macromolecular components. Wheat bran contain a certain amount of ferulic acid, which has a very important influence on the properties of pentosans addition, wheat bran contain a certain amount of uronic acid, this shows that it is an acidic polysaccharide from wheat bran monosaccharide composition analysis, which is mainly composed of arabinose and xylose formed, the ratio between the two types of sugar was 0.63 , and the other contains a certain amount of glucose.

\section{Silty Experiment}

Prepared from wheat bran pentosan crude were $0.2 \%, 0.5 \%, 1 \%, 1.5 \%$ ratio (subject to pentosan content) added to Yu wheat 34 wheat flour and bread flour, and with Brab ${ }^{\circ} \mathrm{Cnd}{ }^{\circ} \mathrm{Cr}$ farinograph (50 g) Effect of pentosan component dough characteristics.

\section{Bread Recipe, Production Process and Evaluation}

Bread Recipe. Application of direct fermentation study impact of pentosan bread baking quality, bread recipe is shown in Table 1 
Table 1 the bread recipe

\begin{tabular}{lc}
\hline Ingredients Content & $/ \mathrm{g}$ \\
\hline Bread flour (wet basis)/\% & 100 \\
Instant dry yeast & 116 \\
Salt & 115 \\
Sugar & 610 \\
Shortening & 310 \\
Water & The optimum amount of water absorption \\
\hline
\end{tabular}

NOTE: The optimal amount of water can be appropriately adjusted according to the amount of water $\mathrm{Brab}{ }^{\circ} \mathrm{Cnd}{ }^{\circ} \mathrm{Cr}$ experiment of silty.

Bread Making Process. Bread making process is as follows: flour and a variety of ingredients weighing y pre-mixing (dough machine, $25^{\circ} \mathrm{C}$ ) y adding an appropriate amount of distilled water, and surface and surface machine 15 miny fermentation $\left(30^{\circ} \mathrm{C}, 80 \%\right.$ relative humidity, $1 \mathrm{~h}$ ) y weighed, cut, split, rounded y proofing $\left(40{ }^{\circ} \mathrm{C}, 80 \%\right.$ relative humidity, $\left.40 \mathrm{~min}\right)$ y baking $\left(220^{\circ} \mathrm{C}, 18\right.$ min) y y cooling bread making bread baking experiments, each times with $500 \mathrm{~g}$ flour experiments, each doing five parallel samples, taken close to three values were averaged.

Bread Quality Evaluation. Loaf volume was determined by rapeseed row weight method, bread specific volume $(\mathrm{cm} 3 / \mathrm{g})=$ volume $(\mathrm{cm} 3) /$ mass $(\mathrm{g})$. Bread score 6 formulated according to the Chinese Academy of Agricultural Sciences 5 bread quality score. 100 points , bread volume 35 points, 5 points surface color, skin texture and shape of bread 5 points, 5 points crumb color, smoothness, 10 minutes, 25 points texture, elastic flexible 10 points, 5 points taste

Bread Aging Test. After the production of bread, natural cooling, sealed into a plastic bag, were measured during storage 0,1,2,3,4,5,6,7 d water hardness and bread crumb of bread.

Crumb Hardness Measurement. Was measured using a rheometer, the rheological conditions: probe diameter of $5 \mathrm{~cm}$ plates, test strength Cheng, $0 \sim 2000 \mathrm{~g}$ assay procedure is as follows: Place the bread in a particular wooden box, cut the bread first two ends of each $2 \mathrm{~cm}$ thick, and then were cut from both ends of the $2 \mathrm{~cm}$ thick slice of bread, unscrewing the central portion of $5 \mathrm{~cm}$ heart bread, bread placed in the heart rheometer test bench above, the probe is lowered, recording crumb is pressed $1 \mathrm{~cm}$ force $(\mathrm{g})$ required when, as an expression of crumb hardness.

Fresh bread soft and delicious, but will harden lose their flavor and texture after a period of time and this is the aging of bread. Bread is generally believed that aging is mainly due to recrystallization of starch that is caused by resuscitation. There is also results show bread moisture content and pentosan content in wheat flour bread aging is affected. With the extension of shelf life of bread, bread hardness increases, bread moisture getting low, so it can be bread hardness changes changes bread and water to represent the degree of aging of bread.

Since wheat bran on bread baking quality 34 powder has a very good improvement effect, this experiment will wheat bran, respectively, $0.2 \%, 0.5 \%, 1.0 \%$ ratio add to Henan wheat flour 34 the study its effect on the bread of aging. As can be seen from the experimental results, during storage of bread, crumb hardness components are stored with the extension of time gradually increased, indicating that during storage of bread, fresh bread gradually aging. With the control group comparison with different proportions of wheat in Henan powder pentosan can effectively slow down the aging bread, when the addition ratio of less than $1.0 \%$, with the increase in the proportion pentosan added, delay staling effect is more significant.

Determination of Crumb Moisture. The measured hardness over crumb crumple, take a certain amount of moisture was measured using crumb direct drying. Crumb moisture content of each group gradually decreased in bread during storage, indicating that during storage of bread, with the storage time, bread dehydration gradually become dry and hard, both bread and gradually aging. Compared with the control group, added pentosan flour can significantly delay the crumb moisture loss, indicating that can effectively delay bread aging. Wheat bran when adding less than $1.0 \%$, with the addition ratio increase, delay staling effect is more significant. 


\section{The Effect of Pentosan from Wheat Bran on Bread Baking Properties}

Pentosan from wheat bran component prepared, respectively, $0.2 \%, 0.5 \%, 1.0 \%, 1.5 \%$ the proportion of bread flour and add to Henan wheat 34 wheat flour, study its effect on bread baking properties

Bread from the experimental results, both for bread flour or wheat flour for yumai 34, when the addition ratio wheat bran $0.5 \%$, the quality of the bread baking good improvement effect, and when the addition ratio of $1.5 \%$ on bread baking quality significantly weakening effect.

Comprehensive farinograghical experiments and bread baking experimental results, wheat bran when the addition ratio was $0.5 \%$, on dough quality and bread baking quality improvement has a very good effect; when the addition ratio was $1.5 \%$ Dough Properties and bread baking quality significantly weakening effect. Wheat bran wherein when the addition ratio was $0.5 \%$, the relatively weak gluten flour quality improvement effect is particularly evident, so the actual application can be considered for wheat bran glycans as flour quality improver, to improve the quality of flour products, especially for relatively weak gluten flour quality improvement.

\section{Conclusion}

Wheat bran under appropriate addition ratio (0.5\%), the dough properties and bread baking quality improvement has a very good role, especially for the improvement of the quality of flour flours relatively weak wheat bran pentosans on dough properties and bread baking quality improvement is mainly reflected in the following aspects: silty water increase the amount of dough, increase dough stability time, thus effectively delay aging bread, wheat bran may be considered pent-poly. as a surface modifier sugar products used in actual production.

\section{Acknowledgements}

Fund Project: Jilin Province Department of Education "The Twelfth Five-Year Guideline" scientific and technological research projects, Kyrgyzstan UNESCO co-word [2014] No. 564

\section{References}

[1] Dougls SD. Cereal Chem, Vol. 6 (2014) No 53, p.25-26

[2] Izydorczyk M S,Biliaderis C G. Cereal Sci, Vol. 12 (2015) No 27, p.74-76

[3] Qian Xiyuan, Jing Jianfen, Hou XuSiem. Food Chemistry, Vol. 30 (2014) No 19, p.144-145

[4] Wang Kuailiang. Food Chemistry, Vol. 29 (2011) No 27, p.21-23

[5] Zhang Gongxu, Sun Jing. Jiangnan University, Vol. 8 (2013) No 27, p.57-60 